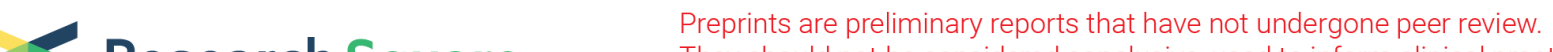 Research Gquare
}

\section{FTO alleviates $A \beta 1-40$ induced retinal pigment epithelium degeneration via PKA/CREB signaling pathway}

YiFan Hu

Shanghai Jiaotong University First People's Hospital https://orcid.org/0000-0002-9332-1852

JieQiong Chen

Shanghai Jiaotong University First People's Hospital

JunRan Sun

Shanghai Jiaotong University First People's Hospital

YuWei Wang

Shanghai Jiaotong University First People's Hospital

PeiRong Huang

Shanghai Jiaotong University First People's Hospital

JingYang Feng

Shanghai Jiaotong University First People's Hospital

Te Liu (D 0721160004@mail.tongji.edu.cn )

Yale University School of Medicine

XiaoDong Sun ( $\nabla$ xdsun@sjtu.edu.cn )

Shanghai Jiaotong University First People's Hospital https://orcid.org/0000-0001-5015-0945

\section{Research}

Keywords: Amyloid- $\beta$, retinal pigment epithelium, RNA methylation, MeRIP, protein kinase A

Posted Date: May 27th, 2020

DOI: https://doi.org/10.21203/rs.3.rs-30549/v1

License: (1) (1) This work is licensed under a Creative Commons Attribution 4.0 International License.

Read Full License 


\section{Abstract \\ Background}

Amyloid- $\beta(A \beta)$, a component of age-related macular degeneration (AMD) hallmark drusen, induces retinal pigment epithelium (RPE) cell degeneration and promotes the progress of AMD. Evidence shows that epigenetics mechanism is involved in the regulation of AMD. In this study, we aimed to investigate the roles of N6-methyladenosine ( $\mathrm{m} 6 \mathrm{~A})$ and its demethylase the fat mass and obesity-associated gene (FTO) in $A \beta$-mediated degeneration.

\section{Methods}

The molecular characteristics and morphology of FTO were examined by quantitative Real-Time PCR (qRT-PCR), Western blot and immunofluorescence. Inhibition of FTO was conducted to analyze its function on cell survival. Ocular Coherence Tomography and Fundus Photography was performed to evaluate the fundus of animal models. m6A-mRNA Epi-transcriptomic microarray, bioinformatics analysis and experimental verification were used to explore the potential molecular mechanism and signaling pathway.

\section{Results}

We found overexpression of FTO in A $\beta$ model and demonstrated that inhibition of FTO by the sodium form of Meclofenamic acid (MA1) aggravated RPE impairment. Mechanistically, we identified protein kinase A (PKA) as FTO's mediating target and found that FTO epigenetically demethylated PKA mRNA and decreased PKA expression, leading to suppressed PKA/ cyclin AMP-responsive element binding (CREB) signaling pathway. Moreover, inhibition of FTO promoted PKA/CREB signaling pathway inducing greater RPE degeneration and death.

\section{Conclusions}

These data demonstrated the functional significance of FTO in Aß-induced RPE degeneration and the regulatory mechanism of PKA/CREB signaling pathway, implying FTO as a potentially therapeutic target for AMD.

\section{Background}

Age-related macular degeneration (AMD) is the leading cause of vision loss among people over 60 . It is estimated that more than 196 million cases of AMD will occur in 2020 [1]. With the advent of antivascular endothelial growth factor (anti-VEGF), the clinical management of this disease have been revolutionized. However, despite advances in the understanding and treatment of AMD in the past 
decade, disease prevalence increases with the ageing human population [2]. A necessity is risen to clarify the pathological mechanism and find out targets for early intervention.

Genetically confirmed that monozygotic twins can present initially with similar early macular pathology, but in the late stages of the disease, they can display different phenotypes [3]. Epigenetic mechanisms may explain some of the phenotypic variability in AMD. Epigenetics regulation includes DNA methylation, histone modification, chromatin remodeling and the regulation of non-coding RNAs [4]. Human AMD samples displayed significant inter-individual variation in RPE transcript expression [5], supporting that heterogenic stress responses underlie AMD pathological process. C57BL/ 6 mice intravitreally injected with Amyloid- $\beta_{1-40}\left(A \beta_{1-40}\right)$ showed degenerative changes and apoptosis within the retina, and microRNAs (mi-RNAs) expression profiling proved differential expression of miRNAs between the $A \beta$ model and the control group [6]. However, these findings cannot fully explain the data obtained from other researches. Since translational repression and mRNA degradation are the outcomes of base pairing between miRNA and mRNA, mRNA modification may also have an impact on AMD pathogenesis [7]. Besides, miRNA regulation is species specific, but mRNA modification is highly conserved in humans and mice, such as N6-methyl adenosine (m6A) RNA methylation. In this way, the mRNA modification mechanisms obtained from mice can be more easily translated into human diseases. Hence, examining post-transcriptional mRNA regulation can better understand epigenetic mechanisms under retinal degeneration and provide a more comprehensive picture of the biology of AMD progression.

N6-methyl adenosine (m6A) RNA methylation is the most abundant internal modifications in RNA, regulating mRNA stability, protein expression, decay and several other cellular processes [8]. Emergent evidence has shown that the m6A modification plays an important role in the occurrence and development of human diseases. m6A levels in mRNA are found to be dynamic, varying during development, aging and in response to cellular stresses [9-10], indicating the role of m6A in age-related degeneration. Fat volume and obesity related (FTO) protein was the first m6A demethylase [11]. It is generally expressed, especially in the brain. Some of FTO variants have impact on neural development that can cause severe growth retardation and diseases like Alzheimer's disease (AD). APOE allelic variant is a significant risk factor for AD [12]. Prospective study showed that FTO interacting with APOE, contributes to AD progression [13]. Interestingly, APOE is also a risk factors of AMD and is related to elevated cholesterol levels and $A \beta$ metabolism [5]. Besides, retina shares the same precursor of the central nervous system (CNS) with brain [14]. With commonalities between AMD and AD of humans and APOE4 transgenic animals [15-16], and the fact that advanced AMD showed an increased risk for developing $A D$ [17], we speculate overlapping molecular mechanism of $A M D$ and $A D$ concerning about APOE4 and $A \beta$ as well as FTO, despite these being two completely distinct diseases.

AMD initially affects the retinal pigment epithelium (RPE), which is a mediator of retinal homeostasis [18]. Impaired RPE causes the formation of extracellular deposits collectively called drusen, which in turn induces RPE degeneration [19]. Increasing evidences support that $A \beta$, a main component of drusen, have played a role in AMD pathogenesis [20]. Evidence shows that $A \beta$ induced cell degeneration via protein kinase A (PKA)/ cyclin AMP-responsive element binding (CREB) signaling pathway [21]. The 
phosphorylation of transcription factor CREB was downregulated in AD models APP/PS1 mouse, indicating $A \beta^{\prime}$ s impact on PKA/CREB signaling pathway [22-23]. However, the activity of PKA in degenerated RPE cells changes under different conditions. While some reported that RPE cells from APP/PS1 mouse showed RPE degeneration and higher enzymatic activation of PKA [24], others suggested that RPE cells from Klotho-knockout mice showed RPE degeneration and lower PKA activation, which downregulated PKA/CREB signaling pathway and led to decreased phagocytosis of RPE, compared with normal human primary RPE cells [25]. This differential activity of PKA in degenerated RPE cells under $A \beta$ treatment may concern about post-transcriptional regulation. Interestingly, FTO was found to alleviate the dephosphorylation of CREB and promote PKA/CREB signaling pathway and BDNF expression [26]. Collectively, above findings point towards a possible association of FTO with RPE degeneration, via $A \beta$ mediated PKA/CREB signaling pathway.

Therefore, we identified the role of FTO in $A \beta_{1-40}$ induced RPE degeneration and investigated the underlying molecular mechanism by which FTO dependent m6A modification affects RPE damage and survival. We first presented evidence that FTO played a protective role in $A \beta_{1-40}$ induced RPE degeneration at the cell and even tissue level. Then mechanistically, FTO was found to selectively demethylate PKA, regulating its mRNA and protein expression, and suppressing PKA/CREB signaling pathway, which partly rescued RPE from degradation. Our results revealed new functions of the m6A mechanism mediated by FTO in RPE damage and survival. We also proposed that targeting retinal epitranscriptome through FTO could be an effective treatment strategy for AMD.

\section{Methods}

Amyloid oligomerization. Lyophilized $A \beta_{1-40}(A 1075$, Sigma) was dissolved in deionized distilled water at a concentration of $1.386 \mathrm{mM}$ and diluted by PBS at a concentration of $350 \mu \mathrm{M}$ according to the instructions. Amyloid- $\beta$ were incubated at $37^{\circ} \mathrm{C}$ for 4 days and stored at $4{ }^{\circ} \mathrm{C}$.

Primary mouse RPE cell isolation and culture. The eyeballs were obtained from 3-week-old C57BL/6 mice. To isolate primary RPE cells, connective tissues of eyeballs were cut off and RPE-choroid complex were separated from entire eyeballs. RPE cells were then gently swapped and transferred in Dulbecco's modified Eagle's medium /Ham's F-12 medium (Gibco) with 25\% fetal bovine serum (Thermo Fisher Scientific) and $1 \% \mathrm{P} / \mathrm{S}$ antibiotics (Beyotime). The cells were cultured at $37^{\circ} \mathrm{C}$ in $5 \% \mathrm{CO} 2$ cell incubator.

Animal model and treatment. To establish $A \beta_{1-40}$ mediated AMD mice, mice were anesthetized with $10 \%$ chloral hydrate, and its pupils were dilated by tropicamide (Santen Oy). A glass micropipette was used to extract $4-5 \mu$ l of vitreous humor from vitreous and deliver $4-5 \mu$ l of $A \beta_{1-40}(175 \mu \mathrm{M})$ into it. Age-matched mice with intravitreally injections (IV) of PBS were regarded as control group.

To establish FTO-suppressing mice, 4-5 $\mu \mathrm{l}$ mixture of $A \beta_{1-40}(87.5 \mu \mathrm{M})$ and Meclofenamate Sodium (S4295, Selleck) $(300 \mu \mathrm{M})$ was intravitreally injected and the control groups were IV-A $\beta_{1-40}(87.5 \mu \mathrm{M})$ mice. Mice with endophthalmitis will be excluded from the analysis. 
Quantitative Real-Time PCR (qRT-PCR). Total RNAs of RPE-choroid tissues or RPE cells were extracted using RNAsimple Total RNA Kit (TIANGEN Biotech) and quantified. Complementary DNAs were synthesized from total RNAs using PrimeScript RT Master Mix (Takara Bio Inc.). Using TAKARA kit to convert RNA to cDNA $\left(37^{\circ} \mathrm{C} 15\right.$ minutes, $85^{\circ} \mathrm{C} 5$ seconds, $4{ }^{\circ} \mathrm{C}$ infinite time).

Quantitative RT-PCR system were performed by a SYBR green-based method and run by a 40-cycle amplification program $\left(95^{\circ} \mathrm{C}\right.$ for $5 \mathrm{~s}, 60^{\circ} \mathrm{C}$ for $30 \mathrm{~s}$ and $72^{\circ} \mathrm{C}$ for $42 \mathrm{~s}$ ) in real-time PCR detection system (Eppendorf). Relative enrichment was calculated using the $2-\Delta \Delta \mathrm{Ct}$ method, and internal controls (GAPDH) were used for normalization. All the primers sequences used in the experiments were listed in Table 1[see Additional file 1].

Western blot analysis. RPE-choroid tissues or RPE cells were lysed in RIPA buffer (Beyotime) containing the complete cocktail of protease inhibitors (Beyotime) and phosphorylase inhibitors (Beyotime). Protein concentrations were determined by BCA protein assay kit (Beyotime). Proteins were separated by 10 or $12 \%$ SDS-PAGE and transferred to polyvinylidene difluoride membranes. The membranes were blocked with blocking buffer (Tris-buffered saline Tween-20 (TBST), containing $5 \%$ nonfat dry milk) for $1 \mathrm{~h}$ at room temperature and blotted with related antibodies at $4{ }^{\circ} \mathrm{C}$ overnight. Secondary antibodies were incubated for $1 \mathrm{~h}$ at room temperature. The membranes were washed 3 times by TBST between each procedure. The membranes with target protein were exposed in visualizer (GE Healthcare). Antibodies were purchased from the following: anti-FTO (1:1000, Abcam), anti-PKA (1:2500, Abcam), anti-p-CREB (1:1000, Cell Signaling), anti-CREB (1:1000, Cell Signaling), anti-BDNF (1:1000, Abcam) and anti-GAPDH (1:1000, Cell Signaling Technology).

Immunostaining. To obtain eyeball tissue slides, the mice were sacrificed after anesthesia and fixed by PFA for 30 minutes. After removing its connective tissues, the eyeballs were extracted, fixed by PFA for 2 hours, and dehydrated by $3 \%$ sucrose aqueous solution overnight. Dehydrated eyeballs were embedded by $\mathrm{OCT}$, frozen at $-80^{\circ} \mathrm{C}$, sliced for observation preparation.

Eyeball tissues were blocked by blocking buffer (PBS, containing $5 \%$ normal goat serum (Beyotime), $0.1 \%$ Triton X-100 (Solarbio) and 3\% $\mathrm{H} 2 \mathrm{O} 2$ ) for $1.5 \mathrm{~h}$ at room temperature and incubated with related antibodies at $4{ }^{\circ} \mathrm{C}$ overnight. Secondary antibodies were incubated for $1 \mathrm{~h}$ at room temperature and tissues were washed 3 times by PBS between each procedure. Nuclei were stained with 4',6-diamidino-2phenylindole (DAPI). Related antibodies were purchased from the following: anti-FTO (1:100, Abcam), anti-PKA (1:100, Abcam), anti-p-CREB (1:800, Cell Signaling), anti-CREB (1:800, Cell Signaling), anti-BDNF (1:500, Abcam) and Alexa Fluor 594-and 488-conjugated secondary antibodies (1:1000, Proteintech). Slides were observed using a fluorescence microscope (Olympus) and a Leica TCS SP8 confocal laser scanning microscope (Leica TCS NT).

Cell proliferation CCK8. RPE cells were seeded at 1000 cells per well in 96-well plates with medium. Cell viability was assayed using Cell Counting Kit-8 (Beyotime) at the concentration in $0,10,50,100,500$, $1000 \mu \mathrm{M} / \mathrm{ml}$, according to operation manual and detected at $450 \mathrm{~nm}$ by Absorbance detector. The results of cell viability were expressed as a ratio (\%) of treated group divided by untreated group. 
Ocular Coherence tomography and Fundus Photography. Each mouse was anesthetized with pupils dilated and placed on a clean plate of OCT/FP detecting equipment (Cold Spring Harbor). The OCT and FP imaging was acquired through the pupil using a scan probe. Pictures were captured and saved in jpg format.

m6A-mRNA Epi-transcriptomic microarray. To perform m6A-mRNA\&IncRNA Epitranscriptomic microarray, total RNAs from RPE-choroid complex of $A \beta$ mice were extracted according to the protocols. Microarray experiments were performed by KangChen Bio-tech, Shanghai, China.

methylated m6A-RNA binding protein immunoprecipitation. RPE-choroid complex tissues were fixed using $1 \%$ methanal for 10 minutes and neutralized by Glycine Solution for 10 minutes at room temperature. After washed twice by PBS, tissues were lysed and incubated with m6A antibody for immunoprecipitation at $4{ }^{\circ} \mathrm{C}$ overnight. Enrichment of m6A containing mRNAs were pulled down by agar beads and washed by PBS twice. After collected, Total RNAs were extracted from beads using RNeasy Mini Kit (QIAGEN) and were converted to cDNA for further qRT-PCR with the primers listed in Above. Enrichment of m6A containing mRNA was analyzed by qRT-PCR with the primer's sequences as follows: PKA, forward 5'ATGGTTATGGAATACGTCCCTGG-3', reverse 5'- AATTAAGAGGTTTTCCGGCTTGA-3';

Statistics Analysis. Each experiment was repeated at least thrice. All the data were analyzed using Graphpad prism 8.0. Multiple t-test were used to compare the statistical difference between related groups. P-values $<0.05$ was considered as statistical significance.

\section{Results}

\section{FTO was increased in A $\beta$ induced RPE degeneration}

To identify whether $A \beta_{1-40}$ treatment alters the expression of m6A regulators in RPE cells, we measured the levels of several major RNAs associated with m6A methylation (METTL3 and METTL14), demethylation (FTO and ALKBH5) and recognition (YTHDF1, YTHDF2 and YTHDF3). The primary mouse RPE cells were cultured with $2.5 \mu \mathrm{M} \mathrm{A} \beta_{1-40}$ for 24 hours. The result of quantitative real-time PCR (qRT-PCR) shown that FTO was up-regulated (2.06 \pm 1.38 -fold) in A $\beta$ group, compared with phosphate-buffered saline (PBS) group (Figure 1a). We further established the intravitreally injected $A \beta_{1-40}$ mice and detected the mRNA level of m6A modification RNAs in the RPE-choroid complex at day 4 (Figure 1b). qRT-PCR data revealed that expression of FTO was increased $(1.53 \pm 0.28$-fold) at RNA levels as compared to its respective controls. To investigate whether $A \beta_{1-40}$ affects FTO protein expression in vivo, FTO expression in RPE-choroid complex was assessed at different time points exposed to $A \beta$ (Figure 1c). In line with initial observation, notable increases of FTO were observed at day 2, day 4 , and day 6 , with the expression peaked at day 4 . Therefore, we chose day 4 for subsequent studies. These findings suggest that $A \beta_{1-40}$ can induce FTO transcription and expression in RPE cells. 
To assess whether the $A \beta_{1-40}$ deposition induces RPE cell impairment, we used fundus photography (FP) to exam the morphology of fundus in $A \beta$ mice at day 4 (Figure 1d). In contrast to vehicle-injected eyes, the fundus of $A \beta$ mice exhibited areas of depigmentation indicating RPE atrophy and underlying photoreceptor degeneration. This finding suggests that $A \beta_{1-40}$ deposition can spread through the retina and induce RPE degeneration. Next, to investigate the potential role of FTO in A $\beta_{1-40}$-mediated RPE degeneration model, we analyzed immunofluorescence staining of $A \beta$-mice retinal sections and found that FTO showed strong fluorescence signal in the nucleus and perinucleus of RPE cells, whereas slight nuclear signaling was observed in the PBS group (Figure 2a). For further confirmation, primary mouse RPE cells exposed to $A \beta_{1-40}$ for $24 \mathrm{~h}$ were analyzed with immunofluorescence staining. FTO fluorescence signal was increased in the $A \beta_{1-40}$ group, and mainly concentrated in the nucleus and perinucleus area, compared with PBS group (Figure $2 b$ ). Collectively, these data established that $A \beta_{1-40}$-induced overexpression of FTO could be an important mediator in RPE degeneration.

\section{Inhibition of FTO exacerbated RPE degeneration in Aß-induced damaging model}

To investigate the beneficial function of FTO during $A \beta_{1-40}$ induced RPE degeneration, we chose the sodium form of Meclofenamic acid (MA-1) as FTO specific inhibitor [27] and measured cell viability of primary mouse RPE cells cultured with different concentration of MA1 at $24 \mathrm{~h}$ (Figure 3a). The cell viability of RPE cells in $50 \mu \mathrm{M}$ MA1 group was $101 \pm 3.5 \%$ and $100 \mu \mathrm{M}$ MA1 group was $97.1 \pm 1.8 \%$, showing no significant difference with PBS group. We further detected FTO expression in RPE cells exposed to various concentrations of MA1 at $24 \mathrm{~h}$ (Figure $3 \mathrm{~b}$ ). We identified that the treatment of $200 \mu \mathrm{M}$ MA1 resulted in significant decreases in the FTO expression levels. Considering the above factors, we chose $100 \mu \mathrm{M}$ MA1 for subsequent studies and validated the inhibition effect of MA1 in A $\beta$-induced damaging model by Western blot (Figure 3c). The protein level of FTO significantly decreased in A $\beta /$ MA1 group, compared with PBS group and $A \beta$ group. Furthermore, we performed intravitreal injection (IV) experiment in $B A L B / c$ mice with a mixture of $87.5 \mu \mathrm{M} A \beta_{1-40}$ and $300 \mu \mathrm{M} M A 1$ as IV-AB/MA1 mice and detected protein level of FTO in its RPE-choroid complex (Figure 3d). Western blot data shown a significant FTOattenuating effect in IV-A $\beta /$ MA1 mice in comparison to IV-A $\beta$ mice. Taken together, our results indicated that MA1 effectively inhibited Aß-induced FTO upregulation in RPE cells.

To clarify the relationship between RPE degeneration and FTO expression, FP and ocular coherence tomography (OCT) were used to assess the fundus and retina of IV-PBS mice, IV-A $\beta$ mice, IV-A $/$ MA1 mice and IV-MA1 mice (Figure 3e). While the fundus of IV-MA1 mice and IV-A 3 mice showed slight and moderate depigmentation comparing to IV-PBS mice, augmented depigmentation was observed in the fundus of IV-A $\beta / M A 1$ mice, indicating that $A \beta$ treatment induced RPE degeneration was aggravated by FTO inhibition. OCT identified no significant changes between IV-PBS mice and IV-MA1 mice. However, IV$A \beta / M A 1$ significantly aggravated retinal structure disorder, compared to IV-A $\beta$, confirming that the inhibition of FTO expression exacerbated RPE degeneration in Aß-induced damaging model. These results suggest that FTO may play a protective role in IV-A $\beta$ mice by rescuing RPE degeneration. 
To investigate the molecular mechanism of FTO and identify its downstream targets in A $\beta$ induced RPE degeneration, we performed m6A-mRNA Epi-transcriptomic Microarray to detect m6A levels of RNA extracted from RPE-choroid complex in A $\beta$ mice and PBS mice. The volcano plot displayed that, $\mathrm{m} 6 \mathrm{~A}$ levels of 571 genes were differentially methylated among 37739 transcripts detected in the microarray (Figure 4a). 78.3\% mRNAs (447 transcripts) were demethylated (green point) and 21.7\% mRNA (124 transcripts) were methylated (red point). Since the increase of other m6A writers and readers could not fully explain the decrease in $\mathrm{m} 6 \mathrm{~A}$, we suggest that FTO overexpression may result in the m6A demethylation in $A \beta_{1-40}$ induced RPE degeneration.

Gene Ontology (GO) analysis report revealed that $A \beta_{1-40}$ induced differential modified m6A RNAs mainly enriched in biological regulation of biological process, binding of molecular function, cell and cell part of cellular component (Figure 4b). Among these, we selected protein kinase A (PKA), a phosphorylase in CAMP/PKA/ cAMP-response element-binding protein (CREB) signaling pathway as a candidate target of FT0-mediated m6A modification for further investigation. Microarray identified that m6A level of PKA was downregulated in $A \beta$ mice, and pathway analysis report suggested that $C A M P / P K A / C R E B$ signaling pathway was involved in regulation of above biological process (Figure 4c). PKA was activated by cAMP level and could phosphorylate CREB, a nuclear transcription factor, to its active state, promoting gene transcription and translation, such as brain derived neurotrophic factor (BDNF) (Figure 4d). To verify m6A level of PKA mRNA in A $\beta$ mice, we performed m6A-RNA binding protein immunoprecipitation (MeRIP). qPCR analyses of MeRIP enriched RNA from A $\beta$ mice confirmed that PKA transcripts were hypomethylated in $A \beta$ mice (Figure $4 \mathrm{e}$ ), suggesting that FTO overexpression may result in PKA mRNA hypomethylation. Taken together, these findings hinted that PKA was a downstream target of FTO.

\section{FTO suppressed PKA transcription and promoted translocation}

To investigate the effect of FTO on PKA expression, we measured FTO and PKA protein level in A $\beta$ mice and IV-A $\beta / M A 1$ mice. While the expression of FTO was significantly increased in A $\beta$ mice, PKA was decreased in comparison to the controls (Figure 5a). When the expression of FTO was inhibited by MA1 in IV-A $\beta /$ MA1 mice, protein level of PKA was reversed, comparing to IV-A $\beta$ mice (Figure $5 b$ ). Later, we confirmed the regulatory effect of FTO on PKA expression in vitro (Figure $5 c$ ). PKA was decreased in RPE cells of $A \beta$ group comparing to PBS group. Meanwhile, the expression level of PKA was higher in $A \beta / M A 1$ group compared to $A \beta$ group. These results show that PKA was significantly suppressed by FTO in ABinduced RPE degeneration model and this suppression could be reversed by MA1 treatment. Next, we used confocal microscopic analysis to detect the immunofluorescence of FTO and PKA expression. FTO and PKA showed significant overlaid fluorescence signal around the nuclei of RPE cells in A $\beta$ mice, whereas limited FTO and PKA signaling was observed in the PBS group (Figure 5d). Strong immunofluorescence of PKA was observed in the nuclear of primary RPE cells exposed to A $\beta$ for $24 \mathrm{~h}$ (Figure 5e), indicating that FTO regulated not only PKA's expression, but also PKA's location. Taken together, these results indicate that PKA expression and translocation can be triggered by FTO overexpression. 


\section{$A \beta$ induced FTO expression regulated PKA/CREB signaling pathway}

To investigate whether FTO mediated PKA/CREB signaling pathway, we detected the mRNA level of FTO and factors of PKA/CREB signaling pathway in A $\beta$ mice (Figure 6a). qRT-PCR showed that FTO expression was increased at mRNA level in A $\beta$ mice, but PKA showed slightly decrease without statistical significance. Downstream targets CREB and BDNF were significantly downregulated. We further evaluated the protein level of FTO and PKA/CREB signaling pathway by Western blot in A $\beta$ mice (Figure $6 b)$. Consistently, a significant increase of FTO was observed, while others were decreased, suggesting that FTO induced PKA down-regulation would result in the suppression of PKA/CREB signaling pathway. To confirm the regulatory role of FTO in PKA/CREB signaling pathway, we established FTO-inhibition model in vivo, and detected the mRNA level of FTO and factors of PKA/CREB signaling pathway (Figure 6c). qPCR data showed that inhibiting FTO reduced the mRNA of FTO and PKA, but promoted mRNA expression of downstream CREB and BDNF, comparing to IV-A $\beta$ mice. We further validated the regulatory effect of FTO through Western blot (Figure 6d). Compared to IV-A $\beta$ mice, suppression of FTO significantly increased the protein levels of PKA, p-CREB and the precursor of BDNF (pro-BDNF), demonstrating that FTO inhibition significantly abolished the suppression of PKA/CREB signaling pathway.

We further validate the effect of FTO on PKA/CREB signaling pathway in vitro. qRT-PCR results of RPE cells from A $\beta$ group showed that FTO was increased at mRNA level as well as PKA and CREB but BDNF showed slightly decrease without statistical significance, comparing to PBS group (Figure 6e). qRT-PCR results of RPE cells from $A \beta / M A 1$ group showed significantly reduction in mRNA levels of FTO, PKA and BDNF, but an increase in CREB, comparing to $A \beta$ group (Figure 6f). Next, we performed Western blot and found that downregulation of FTO caused upregulation of PKA, p-CREB and pro-BDNF (Figure $6 \mathrm{~g}$ ). Taken together, these results indicated that the FTO dependent protective effects on RPE survival and repair was mediated via PKA/CREB signaling pathway.

\section{Discussion}

In this study, we investigated FTO-dependent m6A modification underlying A $\mathrm{B}$ induced RPE degeneration. Treatment with MA1, an FTO inhibitor, aggravated RPE degeneration in A $\beta$ mice. Mechanistically, FTO overexpression downregulated m6A level of PKA mRNA which suppressed PKA/CREB signaling pathway and the expression of downstream target pro-BDNF. FTO alleviated RPE damage at least partially via downregulation of pro-BDNF, a pro-apoptosis gene (Figure 7) [see Additional file 2]. Mediating FTO might be a potential target for AMD treatment.

Major m6A modifying enzymes include methyltransferase, demethylase, and methyl recognition protein. While methyltransferase and demethylase dynamically regulate $\mathrm{m} 6 \mathrm{~A}$ levels, m6A readers recognize m6A sites and facilitate subsequent process [28]. Exploring expression levels of these enzymes will help us understand the overall m6A levels and infer potential pathways in the pathological process. While we selected FTO as our research subject in AB induced damaging model, the mRNAs level of YTHDF1 was increased in vitro and in vivo (Figure 1a and b). As 'm6A reader', YTHDF1 recognize specific m6A mRNA 
sequence and promote mRNA translation [29]. In addition, current study discovered the stimulusdependent characteristics of YTHDF1 [30]. YTHDF1-dependent translation was increased in neurons stimulated by potassium chloride depolarization but showed no alteration before the stimulation. However, this characteristic was restricted in certain cells and YTHDF1's translation was consistently activated in other cells like cancer cells. This finding implied that $A \beta$ may trigger the stimulus-dependent characteristics of YTHDF1, leading to its changeable mRNA level. In our study, we found that over expression of FTO was related to RPE's protection, and suppression of FTO aggravated RPE's degeneration, the above findings show no significant connection with YTHDF1 in the regulation of $A \beta$ induced RPE injury.

MA selectively inhibits FTO by competitively binding with m6A enriched mRNAs. As the sodium form of MA, MA1 still exhibits similarly inhibitory activity on FTO with MA [25]. Despite of its inhibitory function, we observed slight damage in the retina of MA1 mice (Figure 3e). Although earlier studies have reported that high doses of MA may associate with retinal toxicity by inhibiting gap junction conductance of RPE cells [31-32-33], others showed that MA can reduce the inhibition of RPE phagocytosis in acute acrolein and acrolein/hydrogen peroxide combined toxicity models [34], indicating that moderate doses of MA were feasible in the retina. Therefore, we chose $100 \mu \mathrm{M}$ MA1 by CCK8 cell viability test to reduce the side effect of MA1 on RPE cells (Figure $3 a$ and $b$ ). Previous study showed that MA could effectively inhibited FTO's function and expression, influencing its downstream pathways [35]. In our study, we used MA1 to inhibit FTO demethylation in A $\beta$ induced damaging model. We found that FTO inhibited PKA/CREB signaling pathway and downstream target pro-BDNF, and this effect was reversed due to the treatment of MA1.

m6A modifications have multiple effects on mRNA, including mRNA stability, localization, degradation, or translation, which depend on the modified sites of mRNA [36]. From our m6A mRNA-epitranscriptomic microarray analysis, we identified PKA as a candidate of FTO regulation. Our study found that PKA methylation levels were downregulated due to an increase in FTO (Figure 4e). In addition, as PKA gene level slightly decreased without statistic difference, the protein level of PKA was significantly reduced in $A \beta$ mice (Figure 6a and $b$ ), indicating that decreased m6A level of PKA may not directly related to its stability, but to its translation process. Later we found that inhibition of FTO reduced PKA gene level, however, increased PKA protein level in IV-A $/$ MA1 mice (Figure $6 c$ and d), suggesting that decreased FTO may promote the translation of PKA mRNA, which is consistent with previous review. We further confirmed in vitro that decreased m6A level of PKA disturbed translation process in RPE cells (Figure 6e to $\mathrm{g}$ ), but we were unable to clarify the specific mechanism of m6A modification on PKA mRNA, because the m6A-mRNA epitranscriptomic microarray could only recognizes the entire sequence enriched with $m 6 \mathrm{~A}$, such as PKA transcript. Further investigation will be needed to study precise m6A sites on PKA mRNA in order to better understand the mechanism of methylation modification.

As a functional target of PKA/CREB signaling pathway, mature BDNF(m-BDNF) is derived from pro-BDNF after proteolytic cleavage in the extracellular space [37]. The formation rate of $\mathrm{m}-\mathrm{BDNF}$ from pro-BDNF was mediated by the expression of pro-BDNF and tPA- and p11-mediated cleavage process [38]. While 
previous research mainly focused on the neuroprotective factor m-BDNF [39], the pro-apoptosis characteristic of pro-BDNF in AMD are yet fully illuminated. Pro-BDNF binds to $p 75$ neurotrophin receptor (p75NTR) and coreceptor sortilin with high affinity, thereby triggering apoptosis [40]. pro-BDNF dominated over m-BDNF in OXYS mice retina during the development of AMD-like retinopathy, indicating participation of pro-BDNF and the imbalance of neurotrophic factors in the pathogenesis of AMD [37]. The expression of m-BDNF did not change during aging, meanwhile the level of pro-BDNF was significantly increased in the brain of rat[41-42]. We held the opinion that the transformation from proBDNF to m-BDNF was impaired because of the dysfunction of cleavage enzymes in the elderly, resulting in injury aggravating, but we haven't confirmed that yet. Since apoptosis is the major pathologic process for RPE in AMD [43], pro-BDNF play an important role in the progress of RPE degeneration. Besides, pro$B D N F$ increased $A \beta$ level in neuron in vitro, and accelerated $A \beta$ deposition in the brain [44], suggesting that pro-BDNF may play a crucial negative role in the pathogenesis and progression of $A D$ and ageing mice [45], which may also happen in AMD. In our study, we identified pro-BDNF decrease in A $\beta$ induced RPE degeneration. Since downstream target pro-BDNF may trigger apoptosis [46], down-regulated PKA/CREB signaling pathway was in favor of RPE protection. Owing to above results, we suspected that FTO mediated pro-BDNF decrease was beneficial for RPE's survival.

\section{Conclusions}

In summary, we provided evidence that FTO was upregulated in A $\beta$ induced AMD models. High expression of FTO was closely related to protection of RPE by downregulating m6A level of PKA mRNA and PKA expression. Inhibition of FTO aggravated RPE degeneration by activating PKA/CREB signaling pathway. Taken together, our findings suggested that FTO and PKA/CREB signaling pathway may serve as a protective target for AMD.

\section{Additional Files}

Additional file 1. Quantitative RT-PCR primers.xls

Additional file 2. Figure 7 Working schematic.pdf

$A \beta$ induced upregulation of FTO epigenetically demethylated PKA and disturbed its translation, leading to suppressed PKA/CREB signaling pathway and pro-BDNF. Inhibition of FTO reversed the suppression of PKA/CREB signaling pathway and promoted pro-BDNF expression, which aggravated RPE degeneration.

\section{Abbreviations}

$A \beta$, Amyloid- $\beta$; $A D$, Alzheimer's disease; ALKB, alpha-ketoglutarate-dependent dioxygenase; ALKBH5, AlkB homolog 5; AMD, age-related macular degeneration; anti-VEGF, anti-vascular endothelial growth factor; APOE, Apolipoprotein E; APP/PS1, amyloid precursor protein/ presenilin 1; BDNF, brain derived neurotrophic factor; CNS, central nervous system; CREB, cyclin AMP-responsive element binding; DE, 
differential expression; DEG, differentially expressed gene; FP, fundus photography; FTO, the fat mass and obesity-associated gene; GO, gene ontology; IV, intravitreally; m6A, N6-methyladenosine; MA1, the sodium form of Meclofenamic acid; m-BDNF, mature brain derived neurotrophic factor; MeRIP, methylated m6ARNA binding protein immunoprecipitation; METTL3, methyltransferase-like 3; METTL14, methyltransferase-like 14; mi-RNA, microRNAs; OCT, optical coherence tomography; PKA, protein kinase A; pro-BDNF, the precursor of brain derived neurotrophic factor; RPE, retinal pigment epithelium; YTHDF, YT521-B homology (YTH) domain family proteins.

\section{Declarations}

\section{Ethics approval and consent to participate}

All procedures involving mice were according to the Ethics Committee of Jiao Tong University, Shanghai, China, and were fully complied with the resolution on the use of animals in the research of the Association for Research in Vision and Ophthalmology Statement for the Use of Animals in Ophthalmic and Visual Research.

\section{Consent for publication}

Not applicable

\section{Availability of data and materials}

The datasets used and/or analysed during the current study are available from the corresponding author on reasonable request.

\section{Competing interests}

The authors declare no conflicts of interest.

\section{Funding}

This work was supported by the National Natural Science Foundation of China (81730026 and 81700843 ), the National Key Research and Development Program of China (2017YFA0105301, 2016YFC0904800 and 2019YFC0840607), the Science and Technology Commission of Shanghai Municipality (17411953000), Shanghai Sailing Program (19YF1439600, 19YF1439200), Bethune-Langmu eye research fund for the young and middle-aged ophthalmologist (BJ-LM2018001J), National Science and Technology Major Project of China (2017ZX09304010). The funding institutions played no role in the study design, data collection, data analysis, or preparation of this manuscript.

\section{Authors' Contributions}

Y.H., T.L. and X.S. designed the study. Y.H. and J.C. performed all experimental work. J.S., and Y.W. contributed to immunohistochemistry. Y.H. and T.L. performed bioinformatics analysis and data analysis. 
Y.H., J.C., J.S., Y.W., P.H., J.F., T.L. and X.S. contributed to the analysis and interpretation of the data. Y.H. and J.C. wrote the paper. J.C., J.S., T.L. and X.S. contributed to the manuscript revision. All authors read the manuscript and approved the final version.

\section{Acknowledgements}

Microarray experiments were performed by KangChen Bio-tech, Shanghai, China]

\section{Authors' information}

Y.H., Department of Ophthalmology, Shanghai General Hospital, Shanghai Key Laboratory of Ocular Fundus Diseases, Shanghai 200080, China. Shanghai Jiao Tong University School of Medicine, Shanghai 200240, China. J.C., Department of Ophthalmology, Shanghai General Hospital, Shanghai Key Laboratory of Ocular Fundus Diseases, Shanghai 200080, China. Shanghai Jiao Tong University School of Medicine, Shanghai 200240, China. J.S., Department of Ophthalmology, Shanghai General Hospital, National Clinical Research Center for Eye Diseases, Shanghai Engineering Center for Visual Science and Photomedicine, Shanghai engineering center for precise diagnosis and treatment of eye diseases, Shanghai 200080, China. Y.W., Department of Ophthalmology, Shanghai General Hospital, Shanghai Key Laboratory of Ocular Fundus Diseases, Shanghai 200080, China. Shanghai Jiao Tong University School of Medicine, Shanghai 200240, China. P.H., Department of Ophthalmology, Shanghai General Hospital, National Clinical Research Center for Eye Diseases, Shanghai Engineering Center for Visual Science and Photomedicine, Shanghai engineering center for precise diagnosis and treatment of eye diseases, Shanghai 200080, China. J.F., Department of Ophthalmology, Shanghai General Hospital, National Clinical Research Center for Eye Diseases, Shanghai Engineering Center for Visual Science and Photomedicine, Shanghai engineering center for precise diagnosis and treatment of eye diseases, Shanghai 200080, China. T.L., Department of Pathology, Yale University School of Medicine, New Haven 06520, USA. Shanghai Geriatric Institute of Chinese Medicine, Shanghai University of Traditional Chinese Medicine, Shanghai 200031, China. X.S., Department of Ophthalmology, Shanghai General Hospital, National Clinical Research Center for Eye Diseases, Shanghai Key Laboratory of Ocular Fundus Diseases, Shanghai Engineering Center for Visual Science and Photomedicine, Shanghai engineering center for precise diagnosis and treatment of eye diseases, Shanghai 200080, China.

\section{References}

1. Wong WL, Su X, Li X, Cheung CM, Klein R, Cheng CY, et al. Global prevalence of age-related macular degeneration and disease burden projection for 2020 and 2040: a systematic review and metaanalysis. Lancet Glob Health. 2014;2(2):e106-16.

2. Ambati J, Atkinson JP, Gelfand BD. Immunology of age-related macular degeneration. Nat Rev Immunol. 2013;13(6):438-51.

3. Gemenetzi M, Lotery AJ. Epigenetics in age-related macular degeneration: new discoveries and future perspectives. Cell Mol Life Sci. 2020;77(5):807-18. 
4. Lee JT. Epigenetic regulation by long noncoding RNAs. Science. 2012;338(6113):1435-9.

5. Ambati J, Fowler BJ. Mechanisms of age-related macular degeneration. Neuron. 2012;75(1):26-39.

6. Huang P, Sun J, Wang F, Luo X, Feng J, Gu Q, et al. MicroRNA Expression Patterns Involved in Amyloid Beta-Induced Retinal Degeneration. Invest Ophthalmol Vis Sci. 2017;58(3):1726-35.

7. Pillai RS, Bhattacharyya SN, Filipowicz W. Repression of protein synthesis by miRNAs: how many mechanisms? Trends Cell Biol. 2007;17(3):118-26.

8. Zhao BS, Roundtree IA, He C. Post-transcriptional gene regulation by mRNA modifications. Nat Rev Mol Cell Biol. 2017;18(1):31-42.

9. Meyer KD, Saletore Y, Zumbo P, Elemento O, Mason CE, Jaffrey SR. Comprehensive analysis of mRNA methylation reveals enrichment in 3' UTRs and near stop codons. Cell. 2012;149(7):1635-46.

10. Min KW, Zealy RW, Davila S, Fomin M, Cummings JC, Makowsky D, et al. Profiling of m6A RNA modifications identified an age-associated regulation of AGO2 mRNA stability. Aging Cell. 2018;17(3):e12753.

11. Jia G, Fu Y, Zhao X, Dai Q, Zheng G, Yang Y, et al. N6-methyladenosine in nuclear RNA is a major substrate of the obesity-associated FTO. Nat Chem Biol. 2011;7(12):885-7.

12. Liu CC, Liu CC, Kanekiyo T, Xu H, Bu G. Apolipoprotein E and Alzheimer disease: risk, mechanisms and therapy. Nat Rev Neurol. 2013;9(2):106-18.

13. Keller L, Xu W, Wang HX, Winblad B, Fratiglioni L, Graff C. The obesity related gene, FTO, interacts with $\mathrm{APOE}$, and is associated with Alzheimer's disease risk: a prospective cohort study. J Alzheimers Dis. 2011;23(3):461-9.

14. Ohno-Matsui K. Parallel findings in age-related macular degeneration and Alzheimer's disease. Prog Retin Eye Res. 2011;30(4):217-38.

15. Sivak JM. The aging eye: common degenerative mechanisms between the Alzheimer's brain and retinal disease. Invest Ophthalmol Vis Sci. 2013;54(1):871-80.

16. Malek G, Johnson LV, Mace BE, Saloupis P, Schmechel DE, Rickman DW, et al. Apolipoprotein E alleledependent pathogenesis: a model for age-related retinal degeneration. Proc Natl Acad Sci U S A. 2005;102(33):11900-5.

17. Klaver CC, Ott A, Hofman A, Assink JJ, Breteler MM, de Jong PT. Is age-related maculopathy associated with Alzheimer's Disease? The Rotterdam Study. Am J Epidemiol. 1999;150(9):963-8.

18. Golestaneh N, Chu Y, Xiao YY, Stoleru GL, Theos AC. Dysfunctional autophagy in RPE, a contributing factor in age-related macular degeneration. Cell Death Dis. 2017;8(1):e2537.

19. Bruban J, Glotin AL, Dinet V, Chalour N, Sennlaub F, Jonet L, et al. Amyloid-beta(1-42) alters structure and function of retinal pigmented epithelial cells. Aging Cell. 2009;8(2):162-77.

20. Ding JD, Johnson LV, Herrmann R, Farsiu S, Smith SG, Groelle M, et al. Anti-amyloid therapy protects against retinal pigmented epithelium damage and vision loss in a model of age-related macular degeneration. Proc Natl Acad Sci U S A. 2011;108(28):E279-87. 
21. Vitolo OV, Sant'Angelo A, Costanzo V, Battaglia F, Arancio O, Shelanski M. Amyloid beta -peptide inhibition of the PKA/CREB pathway and long-term potentiation: reversibility by drugs that enhance cAMP signaling. Proc Natl Acad Sci U S A. 2002;99(20):13217-21.

22. Gong B, Vitolo OV, Trinchese F, Liu S, Shelanski M, Arancio O. Persistent improvement in synaptic and cognitive functions in an Alzheimer mouse model after rolipram treatment. J Clin Invest. 2004;114(11):1624-34.

23. Ettcheto M, Petrov D, Pedros I, Alva N, Carbonell T, Beas-Zarate C, et al. Evaluation of Neuropathological Effects of a High-Fat Diet in a Presymptomatic Alzheimer's Disease Stage in APP/PS1 Mice. J Alzheimers Dis. 2016;54(1):233-51.

24. He Y, Zhao H, Su G. Ginsenoside Rg1 decreases neurofibrillary tangles accumulation in retina by regulating activities of neprilysin and PKA in retinal cells of AD mice model. $J$ Mol Neurosci. 2014;52(1):101-6.

25. Kokkinaki M, Abu-Asab M, Gunawardena N, Ahern G, Javidnia M, Young J, et al. Klotho regulates retinal pigment epithelial functions and protects against oxidative stress. $\mathrm{J}$ Neurosci. 2013;33(41):16346-59.

26. Lin L, Hales CM, Garber K, Jin P. Fat mass and obesity-associated (FTO) protein interacts with CaMKII and modulates the activity of CREB signaling pathway. Hum Mol Genet. 2014;23(12):3299306.

27. Huang Y, Yan J, Li Q, Li J, Gong S, Zhou H, et al. Meclofenamic acid selectively inhibits FTO demethylation of m6A over ALKBH5. Nucleic Acids Res. 2015;43(1):373-84.

28. Meyer KD, Jaffrey SR. Rethinking m(6)A Readers, Writers, and Erasers. Annu Rev Cell Dev Biol. 2017;33:319-42.

29. Wang X, Zhao BS, Roundtree IA, Lu Z, Han D, Ma H, et al. N(6)-methyladenosine Modulates Messenger RNA Translation Efficiency. Cell. 2015;161(6):1388-99.

30. Shi H, Zhang X, Weng YL, Lu Z, Liu Y, Lu Z, et al. m(6)A facilitates hippocampus-dependent learning and memory through YTHDF1. Nature. 2018;563(7730):249-53.

31. Roy K, Kumar S, Bloomfield SA. Gap junctional coupling between retinal amacrine and ganglion cells underlies coherent activity integral to global object perception. Proc Natl Acad Sci U S A. 2017;114(48):E10484-E93.

32. Ning N, Wen Y, Li Y, Li J. Meclofenamic acid blocks the gap junction communication between the retinal pigment epithelial cells. Hum Exp Toxicol. 2013;32(11):1164-9.

33. Sun H, Wen Y, Ning N, An J, Li J. Retinal toxicity associated with high dose of meclofenamic acid. Drug Chem Toxicol. 2013;36(4):461-5.

34. Sheu SJ, Liu NC, Chen JL. Resveratrol protects human retinal pigment epithelial cells from acroleininduced damage. J Ocul Pharmacol Ther. 2010;26(3):231-6.

35. Zhou P, Wu M, Ye C, Xu Q, Wang L. Meclofenamic acid promotes cisplatin-induced acute kidney injury by inhibiting fat mass and obesity-associated protein-mediated m(6)A abrogation in RNA. J Biol Chem. 2019;294(45):16908-17. 
36. Widagdo J, Anggono V. The m6A-epitranscriptomic signature in neurobiology: from neurodevelopment to brain plasticity. J Neurochem. 2018;147(2):137-52.

37. Telegina DV, Kolosova NG, Kozhevnikova OS. Immunohistochemical localization of NGF, BDNF, and their receptors in a normal and AMD-like rat retina. BMC Med Genomics. 2019;12(Suppl 2):48.

38. Ludka FK, Cunha MP, Dal-Cim T, Binder LB, Constantino LC, Massari CM, et al. Atorvastatin Protects from Abeta1-40-Induced Cell Damage and Depressive-Like Behavior via ProBDNF Cleavage. Mol Neurobiol. 2017;54(8):6163-73.

39. Inanc Tekin M, Sekeroglu MA, Demirtas C, Tekin K, Doguizi S, Bayraktar S, et al. Brain-Derived Neurotrophic Factor in Patients With Age-Related Macular Degeneration and Its Correlation With Retinal Layer Thicknesses. Invest Ophthalmol Vis Sci. 2018;59(7):2833-40.

40. Budni J, Bellettini-Santos T, Mina F, Garcez ML, Zugno Al. The involvement of BDNF, NGF and GDNF in aging and Alzheimer's disease. Aging Dis. 2015;6(5):331-41.

41. Perovic M, Tesic V, Mladenovic Djordjevic A, Smiljanic K, Loncarevic-Vasiljkovic N, Ruzdijic S, et al. BDNF transcripts, proBDNF and proNGF, in the cortex and hippocampus throughout the life span of the rat. Age (Dordr). 2013;35(6):2057-70.

42. Silhol M, Arancibia S, Maurice T, Tapia-Arancibia L. Spatial memory training modifies the expression of brain-derived neurotrophic factor tyrosine kinase receptors in young and aged rats. Neuroscience. 2007;146(3):962-73.

43. Tian L, Kazmierkiewicz KL, Bowman AS, Li M, Curcio CA, Stambolian DE. Transcriptome of the human retina, retinal pigmented epithelium and choroid. Genomics. 2015;105(5-6):253-64.

44. Chen J, Zhang T, Jiao S, Zhou X, Zhong J, Wang Y, et al. proBDNF Accelerates Brain Amyloid-beta Deposition and Learning and Memory Impairment in APPswePS1dE9 Transgenic Mice. J Alzheimers Dis. 2017;59(3):941-9.

45. Chen J, Li CR, Yang H, Liu J, Zhang T, Jiao SS, et al. proBDNF Attenuates Hippocampal Neurogenesis and Induces Learning and Memory Deficits in Aged Mice. Neurotox Res. 2016;29(1):47-53.

46. Teng HK, Teng KK, Lee R, Wright S, Tevar S, Almeida RD, et al. ProBDNF induces neuronal apoptosis via activation of a receptor complex of p75NTR and sortilin. J Neurosci. 2005;25(22):5455-63.

\section{Figures}


a

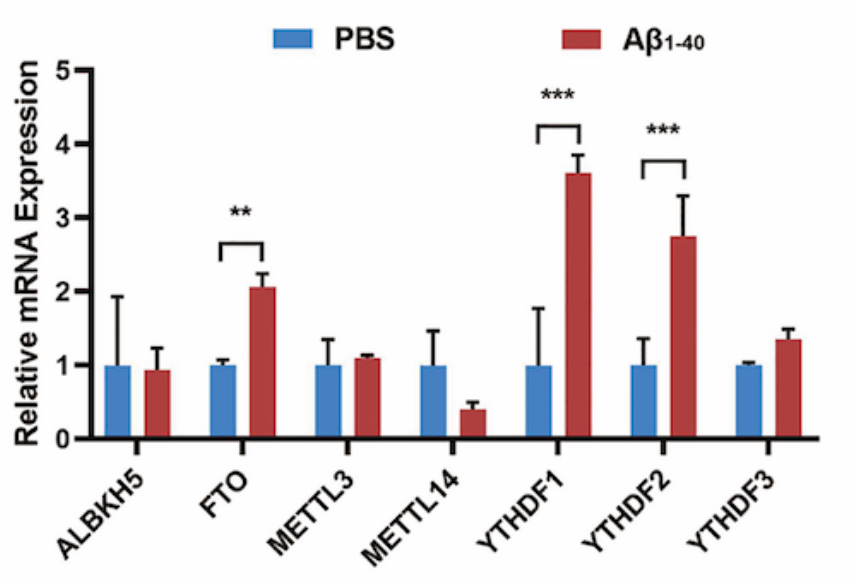

b

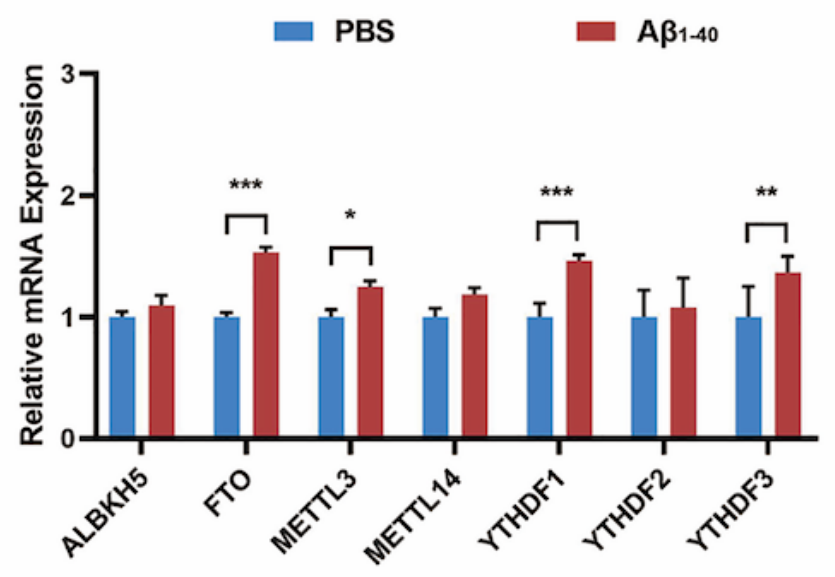

C
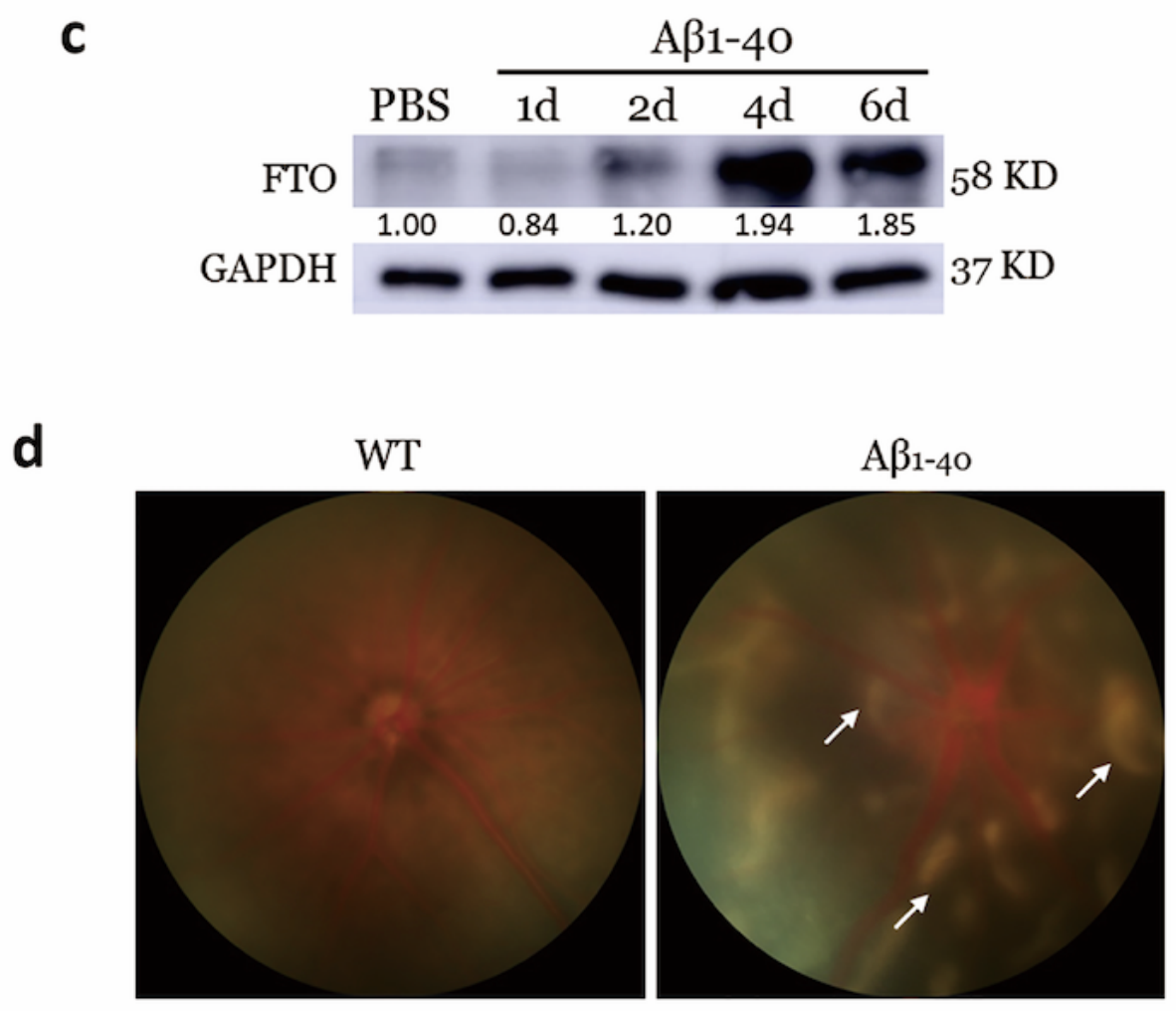

\section{Figure 1}

$A \beta$ induced FTO up-regulation in vitro and in vivo. (a) The primary mouse RPE cells were cultured with $2.5 \mu \mathrm{M} A \beta 1-40$ for 24 hours. Expression of the m6A modification RNAs in primary mouse RPE cells were detected by qRT-PCR. (b) C57BL/6 mice were intravitreally injected with $5 \mu 175 \mu \mathrm{M}$ AB1-40 or PBS. Expression of the m6A modification RNAs in RPE-Choroid complex were detected at day 4 by qRT-PCR. (c) Quantification of FTO expression in RPE-Choroid complex of A $\beta$ mice at day 1, day 2, day 4 and day 6 by Western blot. (d) Fundus of A $\beta$ mice and PBS mice were detected at day 4 by fundus photography (FP). 
The fundus of $A \beta$ mice exhibited areas of depigmentation (arrows) compared with that in PBS mice. Histograms represent the mean and S.E.M. $N=5,{ }^{*} P \leq 0.05 * \star P \leq 0.01,{ }^{\star \star *} P \leq 0.001$ via Student's t-tests.

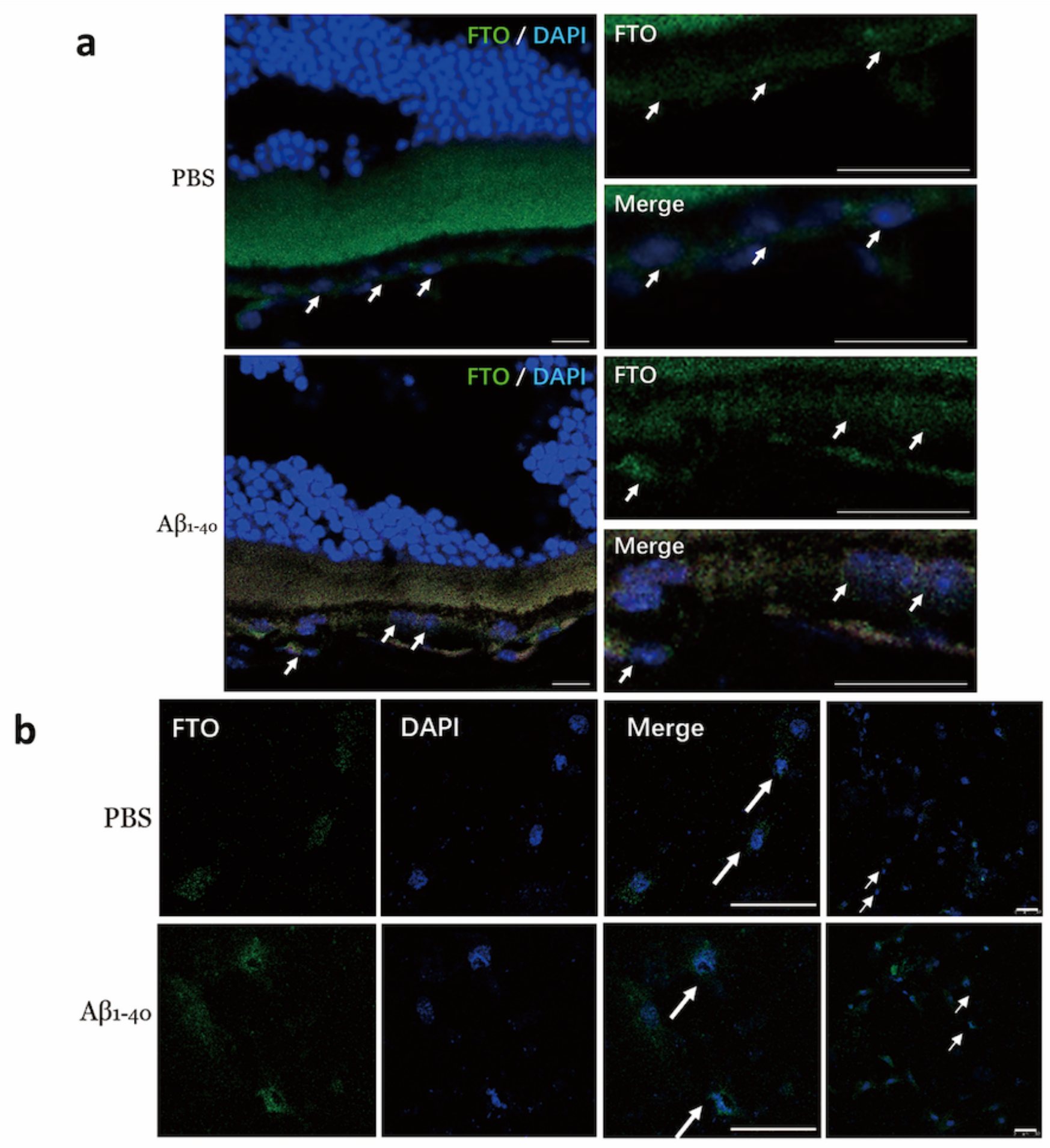

Figure 2

$A \beta$ induced FTO up-regulation expressed in the nucleus and periphery of RPE cells (a) Immunoreactivity for FTO was detected in RPE cell layer (arrows) at day 4 in the A $\beta$-mice retinal sections. Scale bar: $10 \mu \mathrm{m}$. 
(b) Immunoreactivity for FTO was detected in the RPE cells (arrows) in the primary mouse RPE cells exposed to $A \beta 1-40$ at 24 hours. Scale bar: $10 \mu \mathrm{m}$.

a

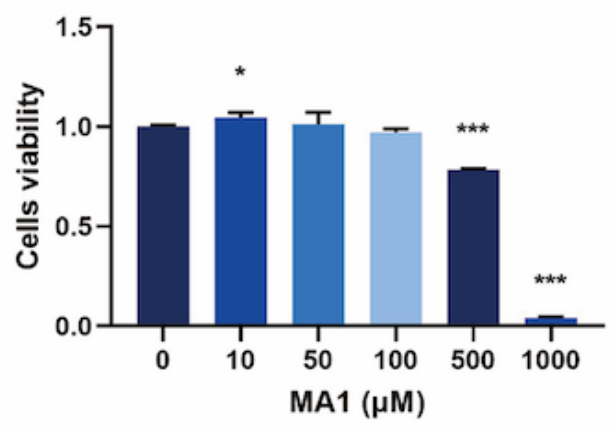

C

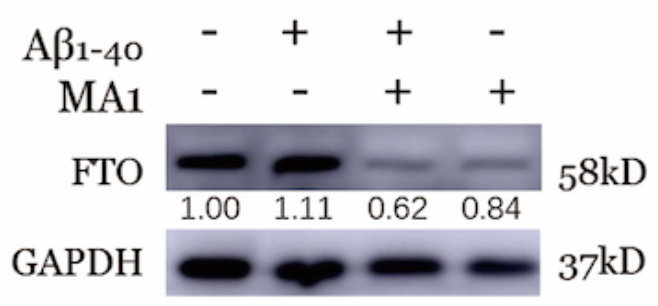

b

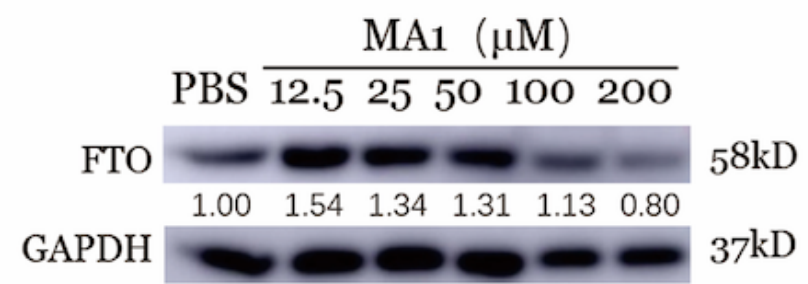

d

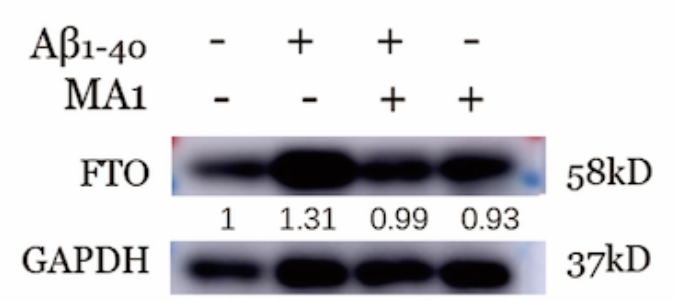

e

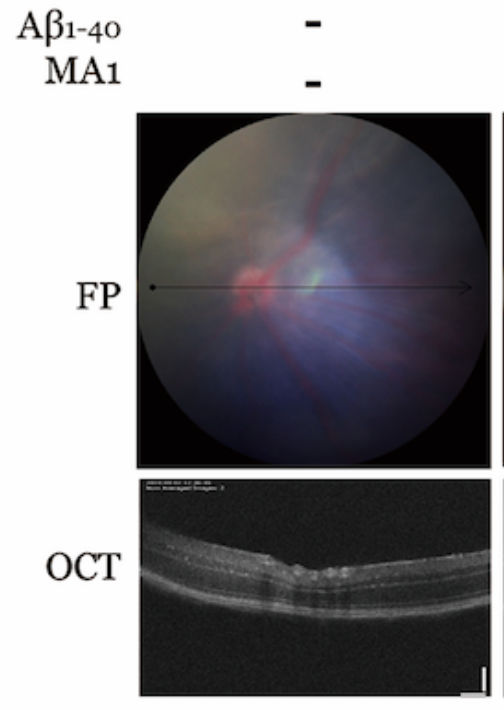

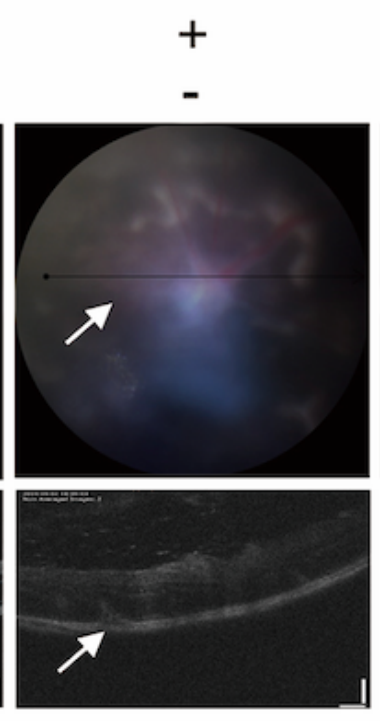
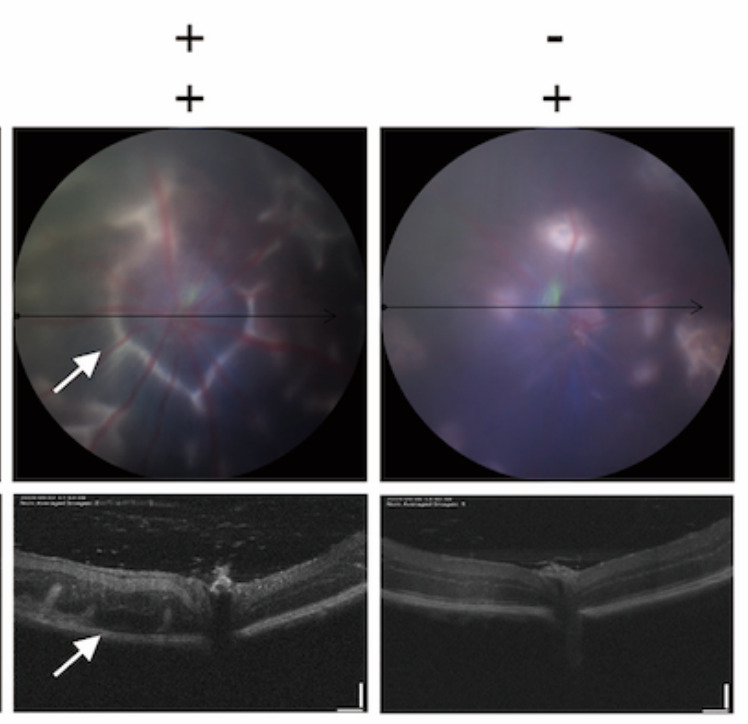

\section{Figure 3}

Inhibition of FTO exacerbated RPE degeneration in A $\beta$ mice. (a) The cell viability of primary mouse RPE cells exposed to different concentration of MA1 were measured by CCK8 assay at 24h. (b) Western-blot showed that a concentration-dependent inhibition effect of FTO expression was induced by MA1 in primary mouse RPE cell at 24h. (c) Western blot assays detected FTO expression in primary mouse RPE cell co-culture with PBS, $2.5 \mu \mathrm{M} \mathrm{A} \beta$, a mixture of $2.5 \mu \mathrm{M} \mathrm{A} \beta$ and $100 \mu \mathrm{M} \mathrm{MA} 1$, and $100 \mu \mathrm{M}$ MA1 at $24 \mathrm{~h}$. (d) 
We performed intravitreal injection (IV) experiment in BALB/c mice with $5 \mu \mathrm{l}$ of $87.5 \mu \mathrm{M}$ A $31-40$ and $300 \mu \mathrm{M}$

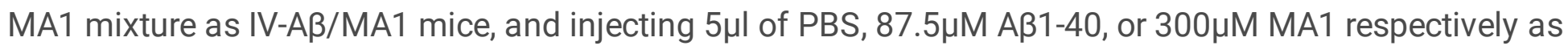
vehicle. Inhibition of FTO protein levels in RPE-choroid complex of IV-PBS, IV-A $\beta$, IV-AB/MA1 and IV-MA1 mice were assessed by western-blot at 4 days. (e) Fundus photography and ocular coherence tomography (OCT) observed that suppression of FTO enlarged hypopigmentation in retina and aggravated retinal structure disorder compared to the control groups. Histograms represent the mean and S.E.M. $N=5, * P \leq 0.05 * \star P \leq 0.01, * \star * P \leq 0.001$ via Student's t-tests.

a

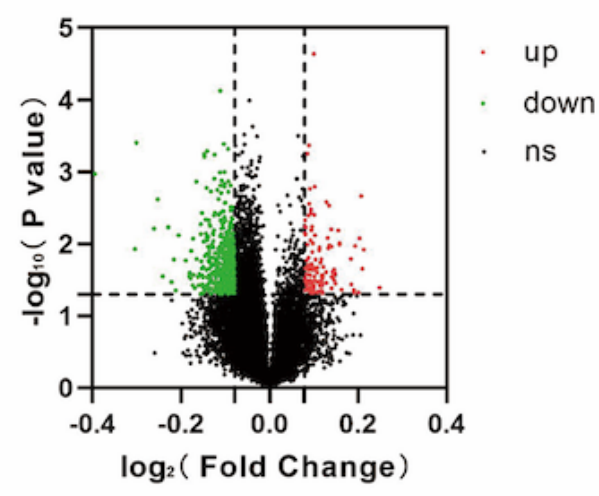

b

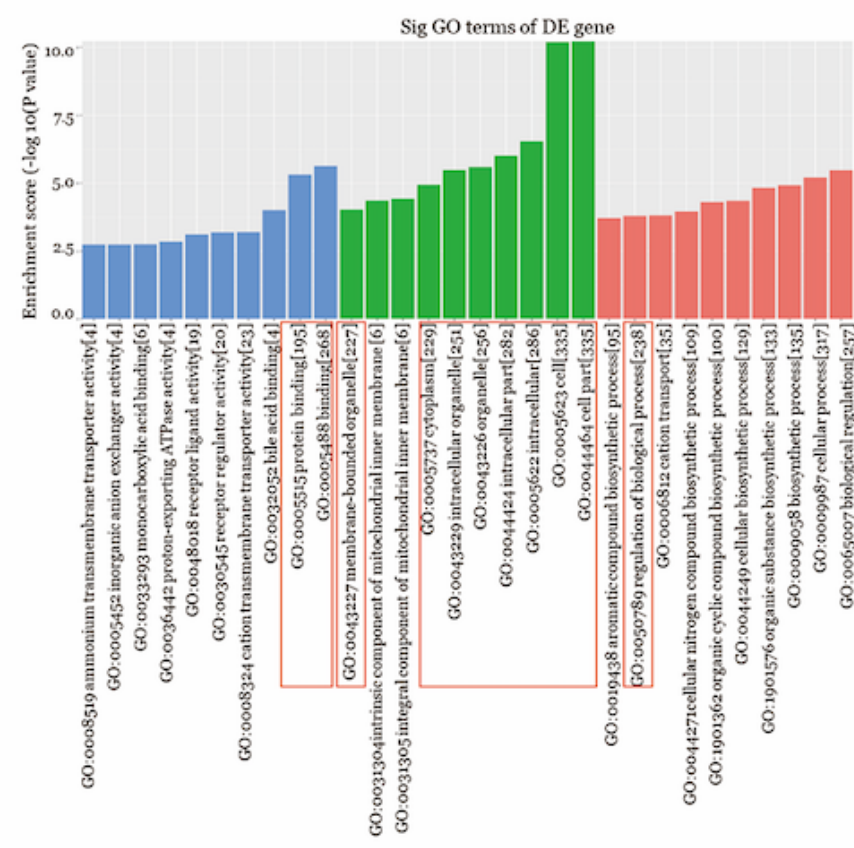

C

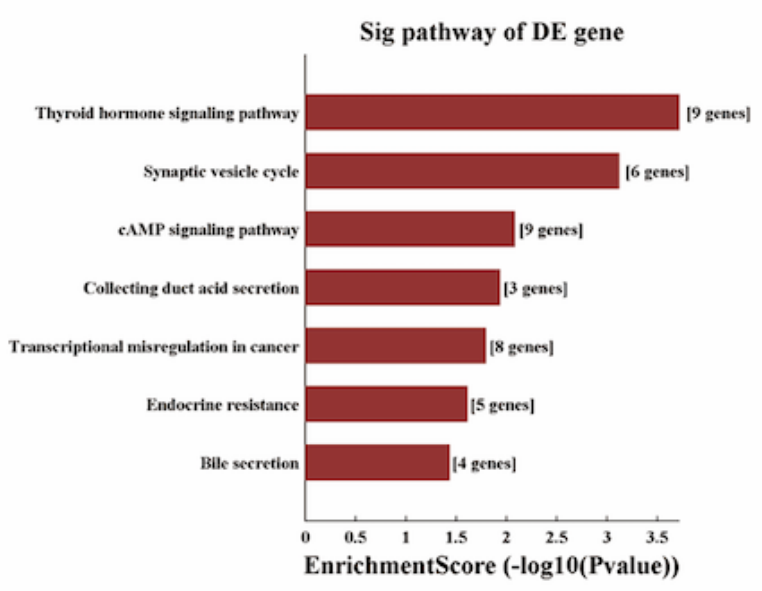

d

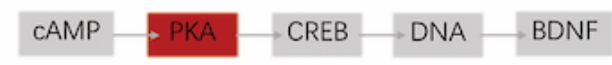

Cell death/survival

e PBS AB1-40

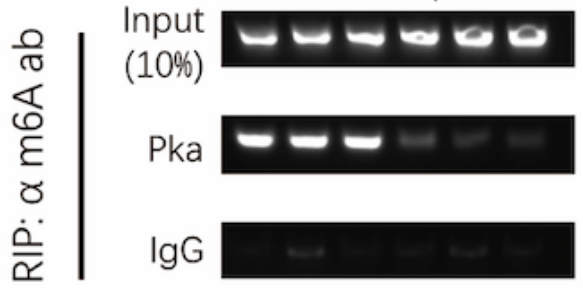

\section{Figure 4}

m6A-mRNA microarray identified PKA as a downstream target of FTO-mediated m6A modification. (a) The volcano plots for the microarray analysis of differential expression in $A \beta$-injected mice and the 
control groups. The $x$-axis is the mean value of log2-fold change, and the $y$-axis shows the -log10 of the $P$ value. The vertical lines represent the cutoff of 1.2-fold change up- or downregulated, and the horizontal line represents the cutoff of $P$ value significance $(P<0.05)$. In this manner, the red points represent the significantly methylated mRNAs and the green points represent the significantly demethylated mRNAs. (b) GO analysis showed that AB1-40 induced differential modified m6A RNAs mainly enriched in cellular process, biological regulation, and regulation of biological process, binding and protein binding of molecular function, cell and cell part of cellular component. The red boxes represent PKA mRNA enriched processes. (c-d) Pathway analysis reported that CAMP/PKA/CREB signaling pathway was involved in regulation of biological process. Red color referred to gene with reduced m6A level. (e) qPCR analyses of m6A-RNA binding protein immunoprecipitation (RIP) confirmed the m6A modification level of PKA mRNA in $A \beta$ mice, compared to PBS mice.

a

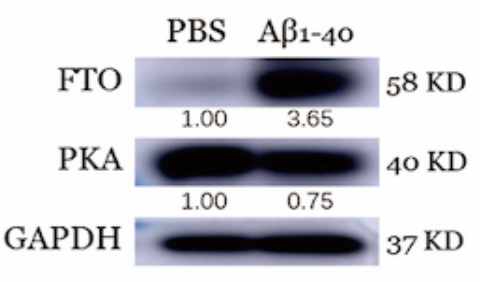

b

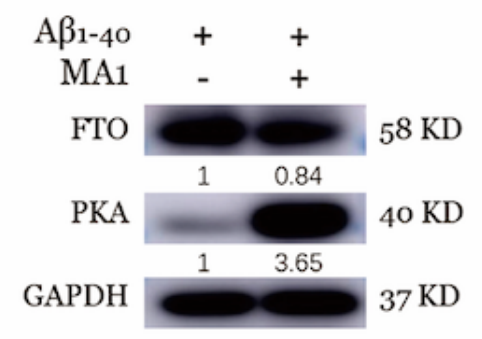

C

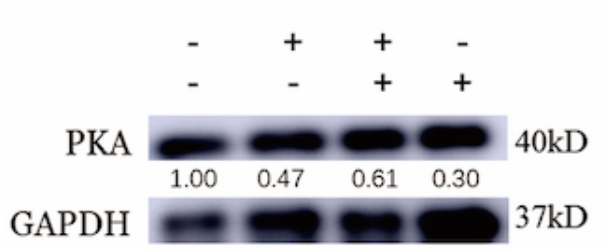

d
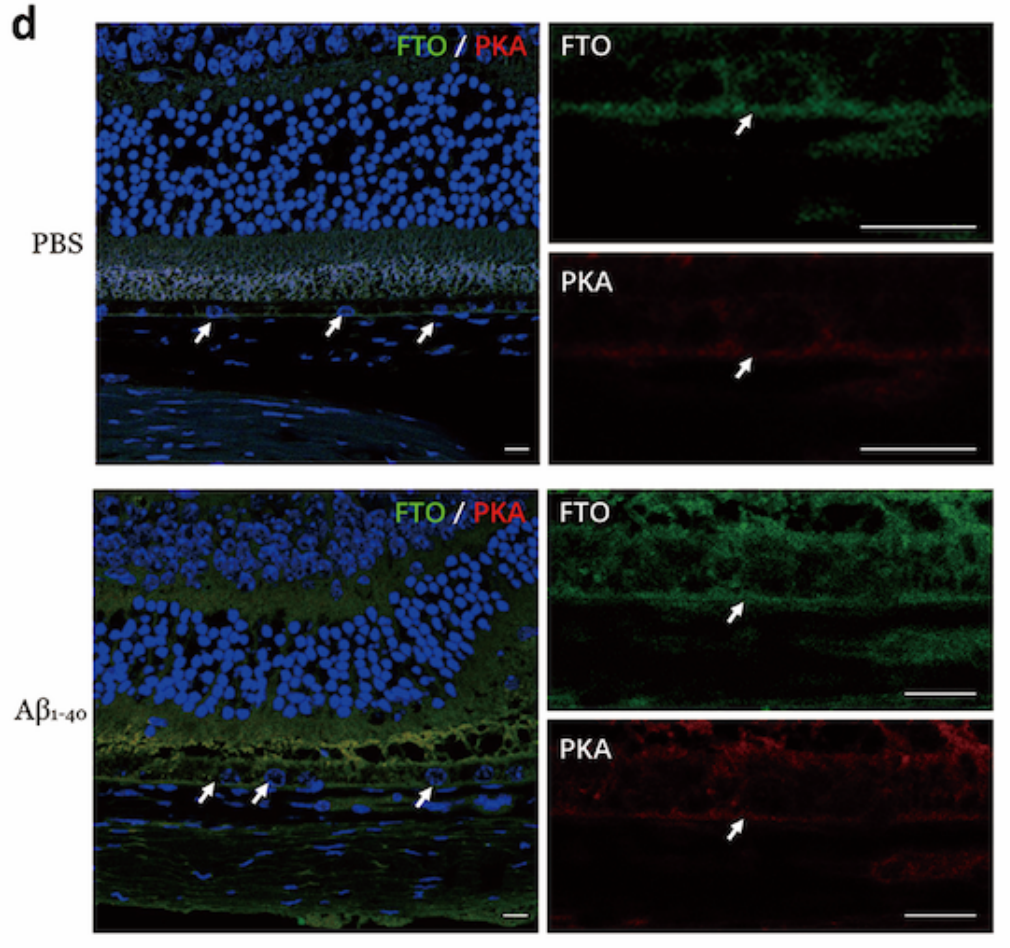

e

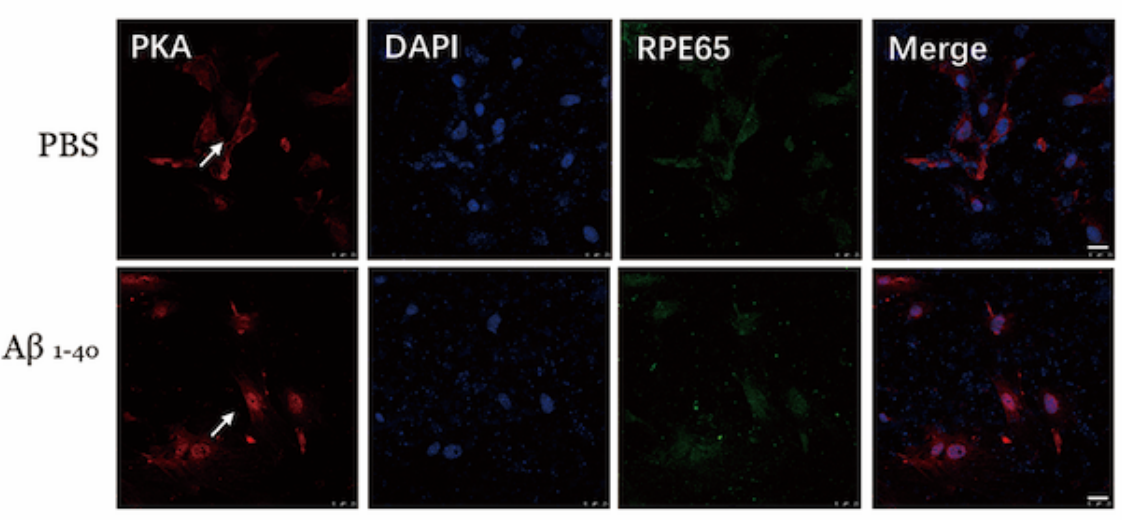




\section{Figure 5}

FTO suppressed PKA transcription and promoted translocation. (a) Western blot assays of FTO and PKA in RPE-choroid complexes in A $\beta$ mice and PBS mice at day 4. (b) Western blot assays of FTO and PKA in IV-A $\beta / M A 1$ mice and IV-A $\beta$ mice at day 4. (c) Western blots showed the protein levels of PKA in primary mouse RPE cells exposed separately to PBS, $A \beta, A \beta / M A 1$ and MA1 for 24h. (d) Retinal sections of $A \beta-$ mice co-immunostained with PKA (red) and FTO (green) were detected in RPE cell layer (arrows) at day 4. Scale bar: $10 \mu \mathrm{m}$. (e) Immunoreactivity for PKA (red) and RPE65 (green)was detected in the RPE cells (arrows) in the primary mouse RPE cells exposed to AB1-40 at $24 \mathrm{~h}$. Scale bar: $25 \mu \mathrm{m}$. 
a

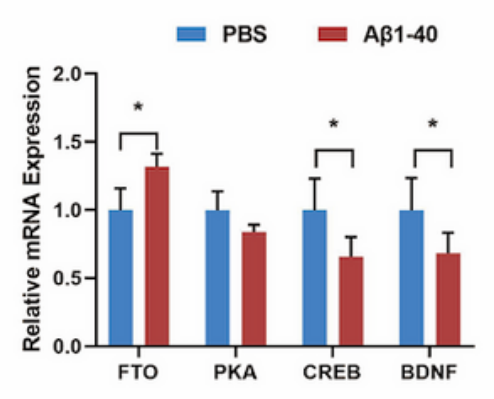

b

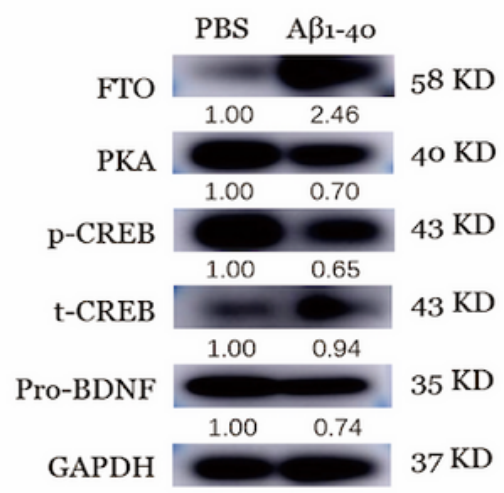

e

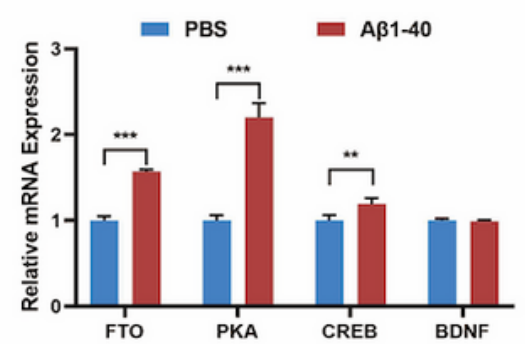

f

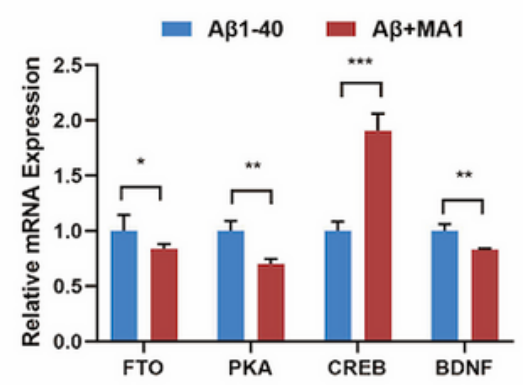

C

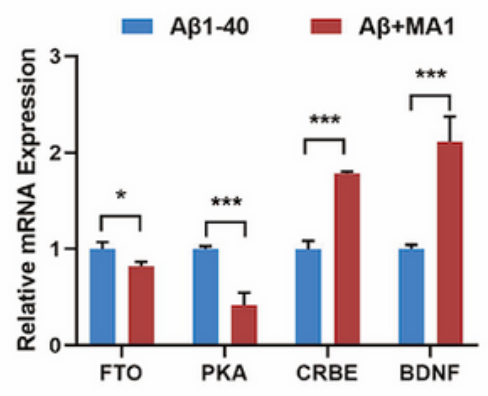

d

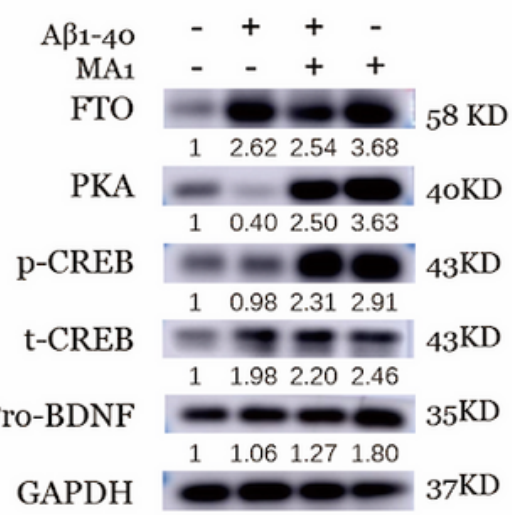

g

\section{Figure 6}

$A \beta$ induced FTO expression regulated PKA/CREB signaling pathway. (a-b) qRT-PCR and Western blots detected the mRNA levels and protein levels of factors in PKA/CREB signaling pathway in RPE-choroid complexes of A $\beta$ mice and PBS mice at day 4. (c) qRT-PCR assays of FTO, PKA, CREB and BDNF mRNAs in RPE-choroid complexes of IV-A $\beta$ mice and IV- AB/MA1 mice. (d) Western blots detected expression levels of factors in PKA/CREB signaling pathway in RPE-choroid complexes of IV-PBS, IV-A $\beta$, IV-AB/MA1 
and IV-MA1 mice at day 4. (e-f) qRT-PCR assays of FTO, PKA, CREB and BDNF mRNAs in primary mouse RPE cells exposed separately to PBS, $A \beta$ or $A \beta / M A 1$ for $24 \mathrm{~h}$. (g) Western blots showed the protein levels of factors in PKA/CREB signaling pathway in primary mouse RPE cells exposed separately to PBS, $A \beta$, $A \beta / M A 1$ or MA1 for 24 h. Histograms represent the mean and S.E.M. $N=5, * P \leq 0.05 * \star P \leq 0.01$, ***P $\leq$ 0.001 via Student's t-tests.

\section{Supplementary Files}

This is a list of supplementary files associated with this preprint. Click to download.

- Additionalfile2.Figure7Workingschematic.pdf

- Huetal.Additionalfile1.table1.xlsx 